\title{
Influence of crops of the cover crop when forming the ridge surface of landings of potatoes to dynamics of parameters of the soil state
}

\author{
A. A. Ustroyev, and E. A. Murzaev
}

Institute of Agroengineering and Environmental Problems of Agricultural Production (IAEP), branch of FSBRI FNATS VIM, St. Petersburg, Russia

\begin{abstract}
The main factors limiting the yield and quality of potatoes are the geometric parameters of the ridge, as well as the parameters of the soil state (moisture, hardness and temperature). In this regard, the task of maintaining the optimal values of these parameters during the potato growing season is urgent. For this, it is proposed, after planting potatoes, simultaneously with the formation of ridges, to sow a cover crop (yellow mustard) on them, followed by its destruction. The purpose of this research is to study the effect of sowing cover crops during the formation of the ridge surface of potato plantings on the dynamics of soil state parameters and potato yield. As a result of the studies, it was found that the use of a new technological method ensures a decrease in the effect of erosion processes on the ridge and the preservation of its geometric parameters, which makes it possible to exclude two inter-row cultivation of potatoes, as well as stabilize the daily soil temperature in the ridge by $2-5^{\circ} \mathrm{C}$ and retain moisture by $5-10 \%$, which allows to increase the yield of potatoes by $11 \%$.
\end{abstract}

\section{Introduction}

The technology of potato cultivation provides, after planting, the formation of full-volume ridges with a fine crumbly soil structure to ensure optimal conditions for the growth and development of plants [1-4]. Such a structure is subject to intense influence of erosion processes and requires hardening of their surface with the help of special technological methods to preserve the volume of the ridges. A patent search and analysis of literary sources [5-7] showed that the most promising method of strengthening the ridge surface can be a technological method of sowing cover crops on it and further destruction of seedlings during the growing season.

The following can be used as cover crops: mustard, oil radish, etc. The short growing season of cover crops allows them to develop a significant amount of green mass and root system within two or three weeks. The created vegetation cover is able to protect the soil surface with its leaf surface, reducing the speed of falling and excluding the ingress of large water droplets on the soil, and also significantly contributes to a decrease in wind speed 
near the earth's surface. All this can lead to a significant reduction or complete elimination of the development of erosion processes in potato plantings [8-10].

Due to the fact that when implementing the proposed technological method, it is necessary to sow cover crop seeds along the ridge surface, seed drills are not suitable for this operation. Fertilizer spreaders can be used as an alternative seeding medium, but such means have a high degree of uneven distribution of the spreading material over the surface of the field.

For sowing cover crops on the surface of the ridges, in our opinion, it is rational to use modern pneumatic seeding modules, which are installed on various agricultural units. Such modules are designed for sowing seeds of various crops and can be installed on various technical means. The module allows to make seeds of agricultural crops both separately and simultaneously with various technological operations performed by basic units [11].

When using standard baffle plates on such systems, the degree of uneven distribution of seeds on the ridge increases under the influence of external factors, for example, wind.

To eliminate this drawback, the existing system for distributing seeds of the pneumatic sowing device APV PS 300 M1 was enhanced by developing special diffusers that were installed instead of baffle plates. The diffuser design is divided into three parts. The first part is the fitting into which the seed tube from the pneumatic sowing device is connected. The second part is the diffuser body itself, inside which a divider is installed. The divider is designed to divide the air flow with the seeds, in order to better distribute it inside the diffuser. The third part is a confuser designed to combine two separated air streams and their orientation to the ridge surface of the field [12].

The purpose of this research is to study the effect of sowing cover crops during the formation of the ridge surface of potato plantings on the dynamics of soil state parameters and potato yield.

\section{Materials and methods}

Experimental studies were performed from June to early July 2020 at the IAEP experimental station in experimental and control versions.

The experiment cultivated potatoes of the "Udacha" variety, zoned for the Leningrad region. The potatoes were cultivated using organic technology without the use of mineral fertilizers and chemical plant protection products. As a cover crop, yellow mustard was used, the sowing of which was performed along the ridge surface, formed simultaneously with deep loosening of the row spacings after planting potatoes on May 28, 2020, using a developed combined unit consisting of a cultivator-deep cultivator for inter-row cultivation of potato plantings of the IAEP design and installed on its APV 300 M1 pneumatic seeder frame with an enhanced distribution system (Figure 1). The cover crop was destroyed on 20 July. Further treatments of potato plantings were not performed in the experiment. In the control variant, pre-emergence harrowing and post-emergence hilling with harrowing were performed. 


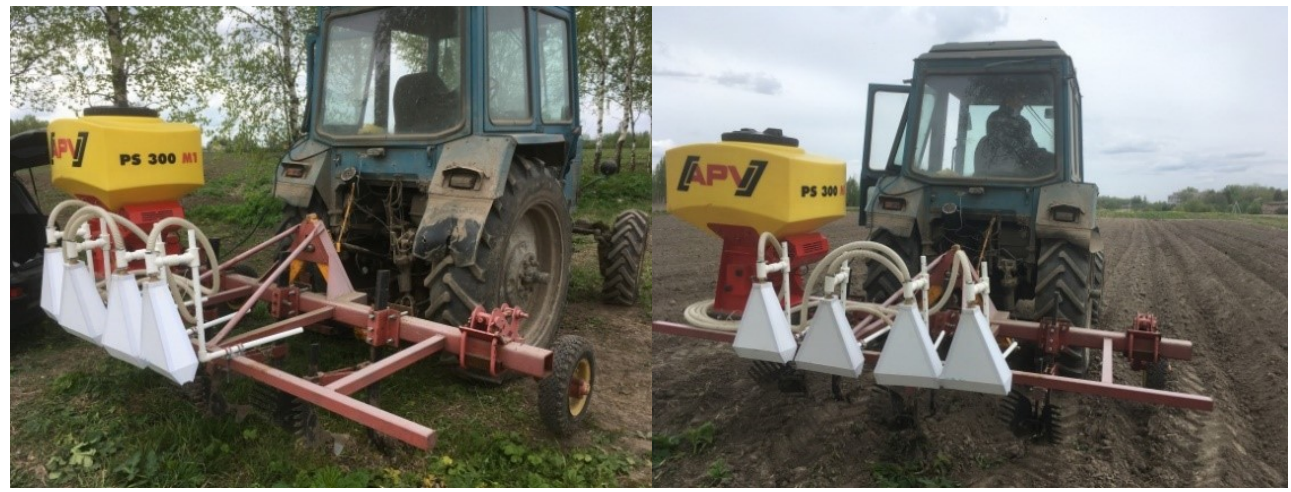

Fig. 1. Multipurpose row-crop cultivator for inter-row cultivation of potato plantings with deep loosening of row spacings and simultaneous sowing of cover crops

A multipurpose row-crop cultivator works as follows. When operating in the field, the deep loosening share on a rigid stand performs deep loosening of the soil in order to eliminate compacted areas in the row spacings formed by the previous passes of the tractor wheels and the planting unit, and also forms favorable conditions for the development of the root system of cultivated crops. Adjustment of the depth of travel of the deep loosening share is achieved by changing the position of the column in height on the frame and can be up to $40 \mathrm{~cm}$. The hilling body behind the hilling body performs the formation of a volumetric loose ridge, directing the soil from the row-spacing to the center of the row with feather dumps. In the course of work, the soil layer is subjected to crumbling, which provides a loose, finely lumpy structure inside the ridge. Seeds are sown into the prepared soil by a pneumatic seeding module through a distributor. The bracket for attaching the diffuser to the machine frame allows to adjust its distance to the profiled surface of the field, the distance from the machine frame to the embedding bodies and the angle of inclination relative to the ridge.

During the experiment, the parameters of the soil state were monitored in the composition: moisture $(\%)$, temperature $\left(\mathrm{C}^{\circ}\right)$, hardness $(\mathrm{MPa})$, geometric parameters of ridges $(\mathrm{cm})$; and potato yield $(\mathrm{t} / \mathrm{ha})$. Soil moisture was measured using a soil moisture meter manufactured by TR di Turuni \& c.Snc, and soil hardness was measured with a penetration logger from Eijkelkamp (Figure 2).

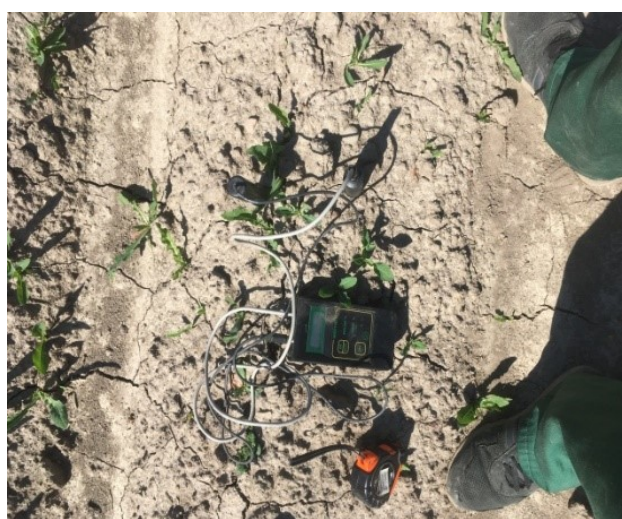

A

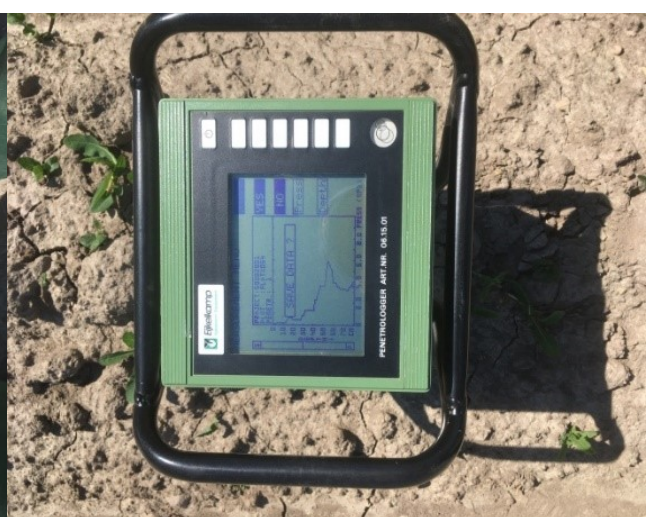

B

Fig. 2. Soil moisture meter manufactured by "TR di Turuni \& c.Snc" (a), penetration logger from Eijkelkamp (b) 
With the aim of a deeper study of the effect of sowing a cover crop on the dynamics of temperature regimes inside the ridges, we have developed an information and measurement system that provides continuous recording of soil temperature in the root layer and the environment over time with a given degree of discreteness (Figure 3).
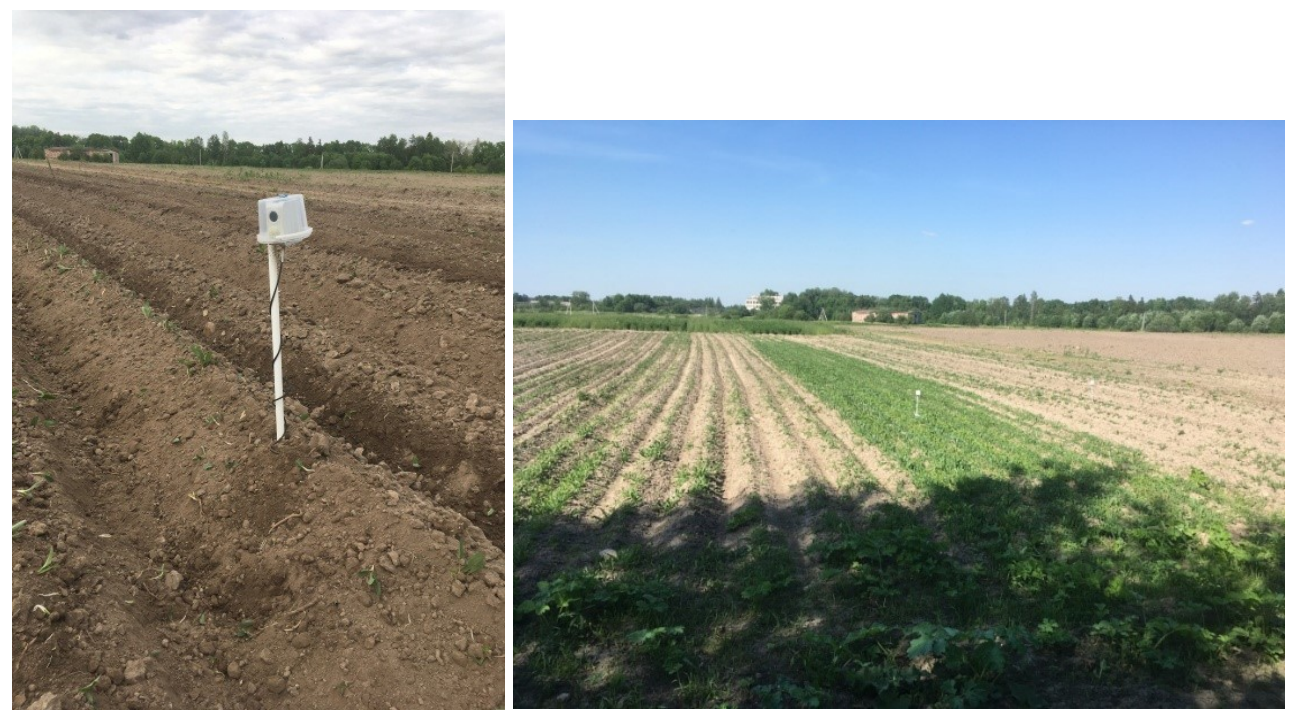

Fig. 3. Information-measuring system of temperature

An information-measuring system consists of several components:

- Debug board based on AVR microcontroller;

- $\quad$ RTC module on DS3231 microcircuit;

- A module for working with a micro SD card;

- Digital temperature sensors $18 b 20$;

- Batteries (3 AAA batteries).

The information-measuring system was made in duplicate and installed at the experimental and control sections. It works pursuant to the software developed by us and accumulates temperature data on a micro SD card in .txt format. For further data processing, Microsoft Excel is used.

\section{Results and discussion}

Table 1 and Figure 4 show the dynamics of the geometric parameters of the ridges of potatoes in the experimental and control versions.

Table 1. Dynamics of the geometric parameters of the ridge, $\mathrm{cm}$

\begin{tabular}{|l|l|l|l|l|l|}
\hline Crest, cm \\
\hline & 15 June & 23 June & 06 July & 21 July & 01 September \\
\hline Experiment & 18.3 & 18 & 18.2 & 17.1 & 16.9 \\
\hline Control & 14.2 & 14.3 & 13.4 & 18.4 & 16.8 \\
\hline
\end{tabular}




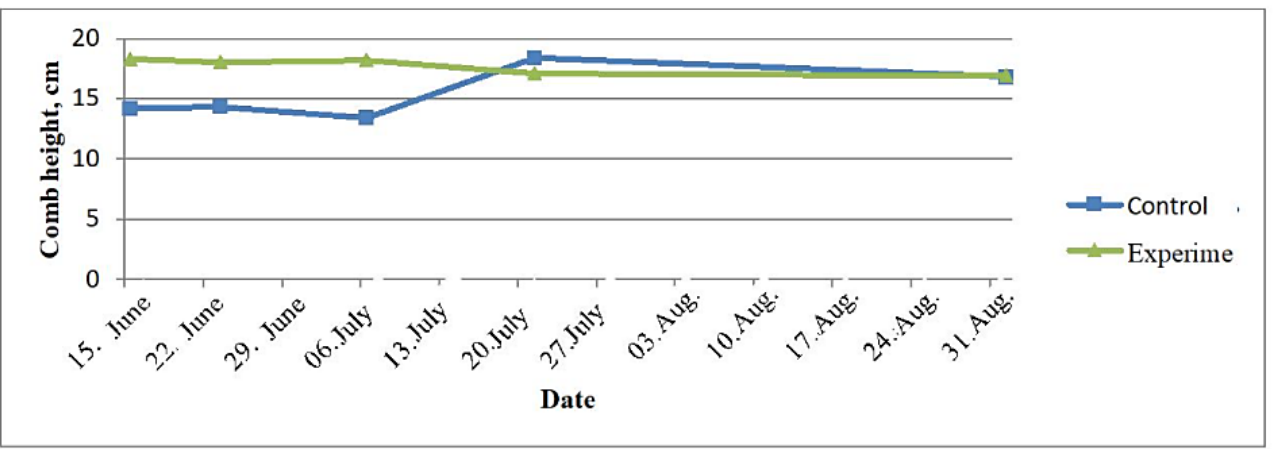

Fig. 4. Dynamics of geometric parameters of potato ridges

Analysis of the data obtained shows that sowing a cover crop on the ridge surface reduced the effect of erosion processes on the ridge, contributing to the preservation of its geometric parameters by $4-7 \mathrm{~cm}$ compared to the control during the growing season of the cover crop. Thanks to this, it was possible to exclude two inter-row cultivation of potatoes.

Figure 5 shows the dynamics of the ambient temperature, the temperature inside the tuberization zone in the control and experimental plots as of June 15.

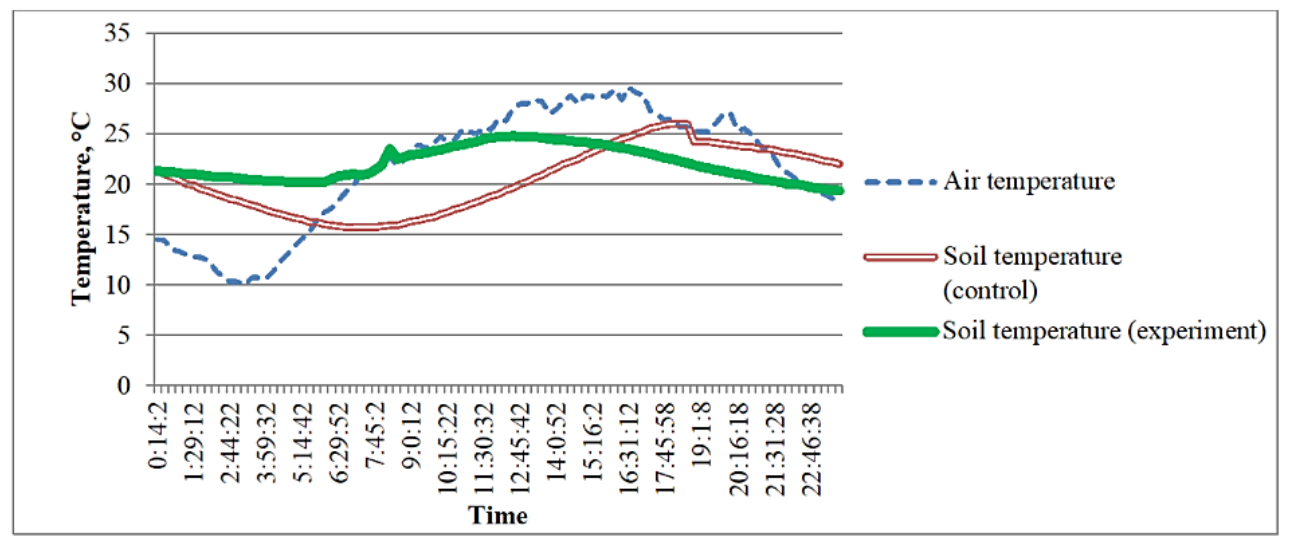

Fig. 5. Daily graph of changes in the temperature of the environment and soil

Analysis of the data obtained shows that with daily fluctuations in air temperature from 10 to $30^{\circ} \mathrm{C}$, the soil temperature in the control ranged from 16 to $26^{\circ} \mathrm{C}$, and in the experiment from 19 to $25^{\circ} \mathrm{C}$. In this way, the cover crop protects the ridge from both overheating and sudden cooling. This trend was observed throughout the entire growing season of the cover crop on the profiled surface. After the destruction of the cover crop, its crop residues continued to restrain the effect of temperature on the ridge until its complete withering away (Figure 6). 


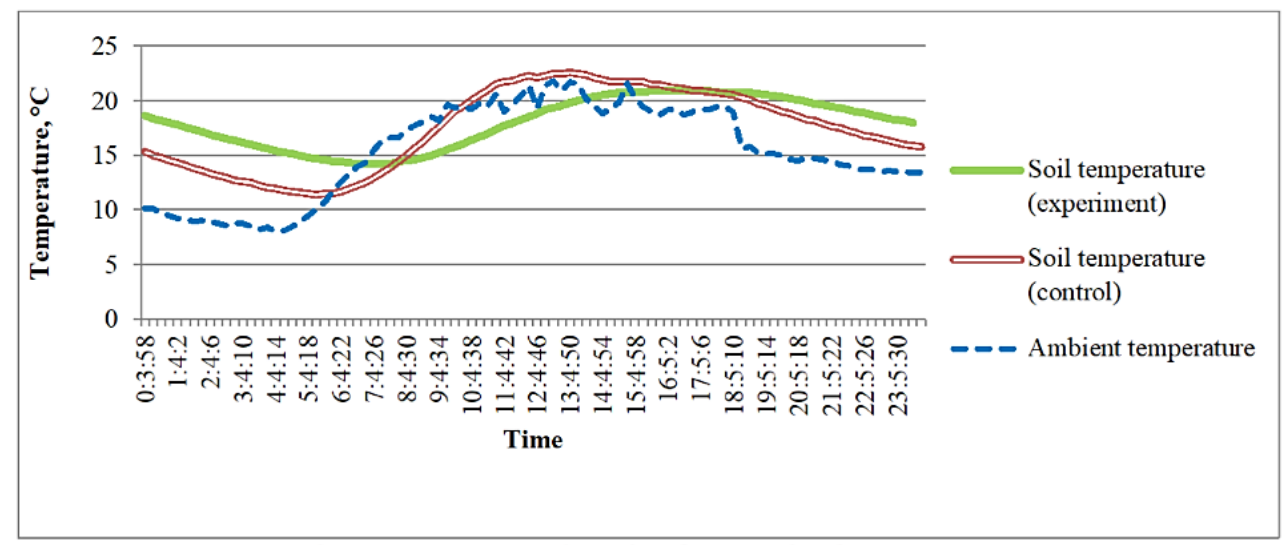

Fig. 6. Temperature dynamics after removing the cover crop

Table 2 and Figure 7 show the dynamics of soil moisture in potato ridges in the tuberization zone in the experimental and control variants.

Table 2. Dynamics of moisture in the ridge in the tuberization zone, $\%$

\begin{tabular}{|l|l|l|l|l|l|}
\hline \multicolumn{7}{|c|}{ Humidity } \\
\hline & 15.06 .2020 & 23.06 .2020 & 06.07 .2020 & 21.07 .2020 & 01.09 .2020 \\
\hline Experiment & 29.18 & 22.9 & 11.56 & 16.98 & 34.3 \\
\hline Control & 22.9 & 29.1 & 22.54 & 16.22 & 36.18 \\
\hline
\end{tabular}

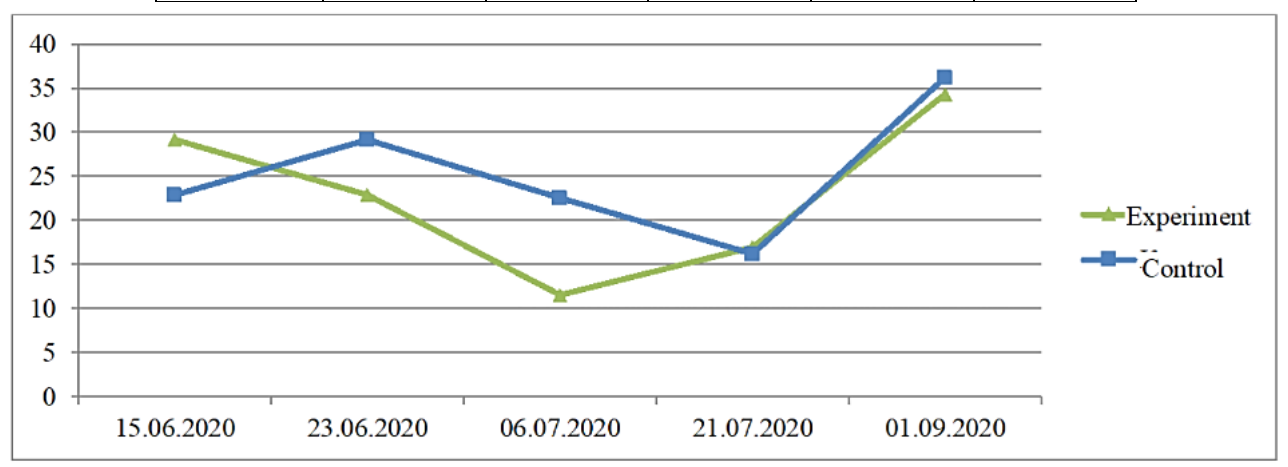

Fig. 7. Dynamics of soil moisture by layers

The analysis of the data obtained shows that the soil moisture as of $06 / 15 / 2020$ in the experiment with the sowing of the cover crop was 5--10\% higher than in the control (without sowing). This is due to the fact that on June 15, the cover crop was in the phase of two leaves, while a "rough soil surface" was created on the ridge and in the aisle, which prevented the free flow of precipitation along the ridge surface, as a result of which a more complete absorption of moisture by the soil took place.

In the course of further growth of the cover crop, the moisture content inside the ridge decreased due to the fact that it, while developing, consumed a large amount of moisture. After the destruction of the cover culture, the humidity was equal in the experiments.

Analysis of the obtained data on soil hardness inside the ridge by layers showed that no significant changes in hardness occurred due to sowing a cover crop on the ridge. 
The average value of the yield of potatoes during experimental research is $18.03 \mathrm{t} / \mathrm{ha}$ in the experiment, which exceeds the value in the control $16.2 \mathrm{t} /$ ha by $11 \%$. This confirms the efficiency of the developed technological method.

\section{Conclusion}

A combined technological method has been developed for processing potato plantings on the ridges in organic cultivation technology using the operations of deep loosening of row spacings and sowing cover crops on the ridge surface with subsequent destruction of seedlings during the growing season.

The use of a new technological method ensures a decrease in the effect of erosion processes on the ridge and the preservation of its geometric parameters, which makes it possible to exclude two inter-row cultivation of potatoes, as well as stabilization of the daily soil temperature in the ridge by $2-5^{\circ} \mathrm{C}$ and moisture retention by $5-10 \%$, which makes it possible to increase potato yield by $11 \%$.

\section{References}

1. V. B. Minin, V. D. Popov, D. A. Maksimov, A. A. Ustroev, S. P. Melnikov, Agronomy Research, 2(18), 1359-1367 (2020)

2. A. B. Kalinin, I. Z. Teplinsky, A. A. Ustroev, P. P. Kudryavtsev, IOP Conference Series: Materials Science and Engineering, 516(1), 1-6 (2019)

3. D. Shpaar, A. Bykin, D. Draeger, Potatoes, 466 (2004)

4. A. B. Kalinin, V. A. Smelik, I. Z. Teplinsky, O. N. Pervukhina, Bulletin of the St. Petersburg State Agrarian University, 39, 315-319 (2015)

5. E. A. Murzaev, Bulletin of the student scientific society of St. Petersburg State Agrarian University, 9(2), 65-68 (2018)

6. A. B. Kalinin, I. Z. Teplinsky, V. A. Ruzhev, World trends and modern technical systems for potato cultivation, 160 (2016)

7. A. B. Kalinin, I. Z. Teplinsky, Rural mechanic, 5, 8-9 (2015)

8. V. D. Abashev, L. M. Kozlova, Agrarian science of the Euro - North - East, 6, 169-178 (2015)

9. M. N. Novikov, A. M. Tamonov, L. D. Frolova, L. I. Ermakova, Agrochemical Bulletin, 4, 20-26 (2013)

10. I. S. Kochetov, Agriculture, 3, 16 (2000)

11. Pneumatic seeding devices, https://www.apvrussia.ru/

12. A. B. Kalinin, A. A. Ustroev, I. Z. Teplinsky, E. A. Murzaev, Technologies and hardware components of the mechanized production of crop production and livestock production, 2(99), 148-157 (2012) 